NOTE TECHNIQUE

\title{
ÉVOLUTION DE LA RÉPARTITION DES ÉCREVISSES EN FRANCE MÉTROPOLITAINE SELON LES ENQUÊTES NATIONALES MENÉES PAR LE CONSEIL SUPÉRIEUR DE LA PÊCHE DE 1977 À 2001
}

\author{
T. CHANGEUX
}

Conseil Supérieur de la pêche, Service Technique, Direction Générale, Immeuble "Le Péricentre ", 16, avenue Louison Bobet, 94132 Fontenay-sous-Bois Cedex, Tél. : +33 (0)1 451436 05, Fax : +33 (0)145143670.

E-Mail : thomas.changeux@csp.environnement.gouv.fr

Reçu 11 août 2003

Accepté le 9 février 2004

Received August 11, 2003

Accepted February 9, 2003

\section{RÉSUMÉ}

Selon les enquêtes nationales menées par le Conseil supérieur de la pêche depuis 1977 en France métropolitaine, la répartition des espèces d'écrevisses montre que les espèces autochtones (Austropotamobius pallipes, Astacus astacus, Austropotamobius torrentium) sont soit rares, soit en forte régression, alors que les espèces introduites (Orconectes limosus, Pacifastacus leniusculus, Procambarus clarkii, Astacus leptodactylus) progressent. L'interdiction de transport vivant des espèces introduites, à l'exception d'A. leptodactylus, n'a pas enrayé le phénomène avec une accélération particulière de la progression de $P$. leniusculus ces cinq dernières années, certainement responsable de la recrudescence des cas de peste (Aphanomyces astaci).

Les autres mesures favorisant les espèces autochtones, comme la limitation des prélèvements par pêche et les programmes de réintroduction, n'inversent pas la tendance. II semble donc nécessaire de développer une politique de conservation centrée sur la préservation de l'habitat et le respect de certaines règles de prophylaxie à déterminer. Cela ne peut s'envisager que sur des bassins versants de petite surface facilement contrôlables s'il existe un fort engagement des autorités locales. Les 55 arrêtés de biotope, et surtout les 154 sites Natura 2000 avec leurs plans de gestion associés, apparaissent comme des voies prometteuses pour mettre en œuvre ce type d'actions en France. Le cas de l'île Corse, récemment colonisée par $O$. limosus, mériterait des mesures réglementaires particulières pour éviter que $P$. leniusculus et $P$. clarkii, ne la suivent dans l'avenir.

Mots-clés : écrevisses, répartition géographique, variation temporelle, gestion, conservation, France.

\section{CHANGES IN CRAYFISH DISTRIBUTION IN METROPOLITAN FRANCE ACCORDING TO THE NATIONAL SURVEYS PERFORMED BY THE CONSEIL SUPÉRIEUR DE LA PÊCHE FROM 1977 TO 2001}

\author{
ABSTRACT \\ When looking at the various national surveys performed in Metropolitan France since \\ 1977 by the Conseil supérieur de la pêche, crayfish species distribution shows that native
}


species (Austropotamobius pallipes, Astacus astacus, Austropotamobius torrentium) are either rare, or in decline; while the introduced species (Orconectes limosus, Pacifastacus leniusculus, Procambarus clarkii, Astacus leptodactylus), are increasing. The ban of live introduced species transport, except for A. leptodactylus, didn't stop this process with a peculiar acceleration of $P$. leniusculus propagation these last five years, certainly responsible for plague (Aphanomyces astaci) renewed outbreaks.

The other measures in favor of native species, such as fishing limitations and reintroduction plans, have not reversed the trend. So, it seems necessary to develop a conservation policy based on habitat preservation and on the respect of certain prophylactic rules which are to be determined. That can only be considered on watersheds of small area, easily controlled if local authorities are involved. The 55 French habitat decrees, and the 154 Natura 2000 zones with their management plans, seem to be a promising way to implement this type of actions in France. The case of Corsica, recently colonized by $O$. limosus, would require special measures to prevent the island from invasion by $P$. leniusculus and $P$. clarkii in the future.

Key-words: crayfish, geographical distribution, temporal variation, management, conservation, France.

\section{INTRODUCTION}

Les écrevisses tiennent en France et en Europe une place particulière. Spécialement appréciées pour leur chair, ce sont les seuls crustacés d'eau douce faisant l'objet d'une exploitation ciblée par pêche. C'est pourquoi elles sont assimilées aux poissons dans le code de l'environnement (Art. L. 431-2 et arrêtés d'application) et leur pêche fait l'objet d'une réglementation distincte (Art. R. ${ }^{*} 236-30,32$ et 34).

Sept espèces d'écrevisses ont été identifiées en France : trois espèces autochtones et quatre espèces introduites. Les trois espèces autochtones occupaient à l'origine tous les habitats disponibles : l'écrevisse à pieds blancs (Austropotamobius pallipes) généralement dans les rivières de la zone à truite, l'écrevisse à pieds rouges (Astacus astacus) dans les parties plus aval, et l'écrevisse des torrents (Austropotamobius torrentium) dans quelques petites rivières du Nord-Est du pays. Ces espèces sont actuellement classées vulnérables par l'International Union for Conservation of Nature and Natural Resources (UICN ${ }^{1}$ ). Comme de nombreuses espèces d'eau douce, les écrevisses autochtones sont menacées par la dégradation de leur habitat ainsi que par les pollutions. Mais elles se singularisent surtout par une très grande sensibilité aux introductions d'autres espèces d'écrevisses, vectrices de maladies. A ce titre, elles peuvent bénéficier de dispositions réglementaires spéciales présentées par VIGNEUX et al. (2002), concrétisées par les arrêtés du 21 juillet 1983 et du 18 janvier 2000.

Parmi les quatre espèces d'écrevisses introduites, trois espèces sont issues d'Amérique du Nord: l'écrevisse américaine (Orconectes limosus) est originaire de la côte Est des Etats-Unis, l'écrevisse signal (Pacifastacus leniusculus) vient de Californie et l'écrevisse de Louisiane (Procambarus clarkii) est issue des bayous du Mississippi. Toutes ces espèces du Nouveau monde sont porteuses saines de la peste de l'écrevisse (Aphanomyces astaci) ou aphanomycose, une maladie capable d'éradiquer une population d'écrevisses autochtones en quelques semaines. En outre, certaines d'entre-elles ont un impact sur le milieu (creusement) et rentrent en compétition avec les espèces autochtones. Elles ont donc été classées dans la liste des espèces susceptibles de provoquer des déséquilibres biologiques (Art. R. ${ }^{*} 232-3$ ), espèces pour lesquelles il a été pris des

(1) http://www.redlist.org 
mesures dans l'objectif de limiter leur dissémination (introduction interdite (Art. L. 43210) et transport vivant interdit (Art. L. 432-11)). L'écrevisse à pattes grêles (Astacus leptodactylus), quatrième espèce introduite, est originaire d'Asie mineure et centrale. Au même titre que les espèces autochtones, elle est sensible à la peste de l'écrevisse et n'est donc pas porteuse saine de cette maladie. Intégrée à la liste des espèces présentes en France (Arrêté du 17 décembre 1985 pris en application de l'article L. 432-10), elle peut être introduite dans toutes les eaux douces.

Les enquêtes nationales menées auprès des brigades du Conseil Supérieur de la Pêche (CSP) en 1977, 1988 (publiée dans l'Atlas des crustacés décapodes d'eau douce, VIGNEUX et al., 1993) et 1995 ont montré que l'évolution de la répartition des écrevisses était très rapide. Cette situation a suscité la mise en place d'une veille écologique particulière à l'échelon national (ARRIGNON, 1990 ; CHANGEUX, 1996), régional (réseaux AuvergneLimousin, Bourgogne, Rhône-Alpes, Poitou-Charentes) et départemental (départements : $11,17,21,22,33,34,37,51,52,61,64,70,72,79,83 \ldots)$ ou bien encore attachée à des parcs naturels (Haut Languedoc, Lacs de la Forêt d'Orient...) et des réserves. Le présent document rend compte de l'enquête nationale menée en 2001 auprès des brigades du CSP, six ans après la dernière enquête qui date de l'été 1995.

\section{MÉTHODE}

Un questionnaire d'enquête "express écrevisses ", semblable à celui employé en 1995, a été mis en place par la mission informatique sur le réseau intranet du CSP à la fin de l'été 2001, de manière à couvrir la saison de pêche des écrevisses.

Le questionnaire à remplir par chaque brigade (voir copie en annexe 1), porte sur l'ensemble du département sans faire intervenir de localisation plus détaillée. Chacune des cartes, présentées dans la suite, résume les informations concernant aussi bien les eaux libres (cours d'eau, lacs et retenues de $1^{\text {ère }}$ et $2^{\text {ème }}$ catégorie) que les eaux closes et les piscicultures. Les présences résultant simplement d'opérations de retrempage ont été exclues tant qu'aucune preuve d'acclimatation n'était fournie. L'évolution à court terme a été appréciée en distinguant 5 classes selon la situation inter et intrapopulationelle : (1) disparition de population, (2) maintien des populations avec diminution des effectifs, (3) maintien des populations et des effectifs, (4) maintien des populations avec augmentation des effectifs, (5) apparition de populations. L'abondance a été évaluée à partir du pourcentage de linéaire occupé ou de l'occurrence dans les plans d'eau du département.

Pour suivre l'évolution à long terme de la répartition, espèce par espèce, des cartes semblables ont été réalisées à partir des enquêtes précédentes (1977, regroupement des références 1988 à 1990 sous l'année 1990, et enquête " express écrevisses " 1995). Les protocoles adoptés au cours des différentes enquêtes ont été suffisamment semblables pour qu'elles soient comparables entre elles. Ces indications sont utilisées dans la suite pour parler d'apparition ou de disparition, de diminution ou d'extension. Toutefois, comme c'est généralement le cas pour les informations de répartition, si la présence d'une espèce (figurée en grisé) est une donnée avérée, l'absence (figurée en blanc) est une information moins définitive car il n'est pas possible de mettre en œuvre une prospection intégrale des milieux aquatiques.

En complément, l'utilisation des possibilités réglementaires de protection a été mesurée (1) au travers des sites Natura $2000^{2}$; (2) à partir des arrêtés de biotope, faisant référence au nom d'une des trois espèces d'écrevisses autochtones ${ }^{3}$.

(2) Tel que référencés sur http ://natura2000.environnement.gouv.fr/especes/IDX2.html

(3) Selon la base de données du service du patrimoine naturel au Muséum National d'Histoire Naturelle. 


\section{RÉSULTATS}

Les résultats de l'enquête sont consignés dans les tableaux de contingence donnés en annexe 2. Contrairement aux résultats de 1995, les sept espèces sont toutes répertoriées, y compris l'écrevisse des torrents, récemment identifiée dans le département de la Moselle (MACHINO, 1995 ; MACHINO, 1996 ; FRANCKHAUSER et MACHINO, 2001). La situation des trois espèces autochtones (A. pallipes, $A$. astacus, $A$. torrentium), et des quatre espèces introduites (O. limosus, $P$. leniusculus, $P$. clarkii, $A$. leptodactylus) est examinée dans la suite.

\section{Espèces autochtones}

\section{L'écrevisse à pieds blancs (Austropotamobius pallipes)}

Cette espèce est considérée comme la principale espèce autochtone. Dans ce groupe, c'est effectivement l'espèce la plus répandue sur l'ensemble du territoire métropolitain. Elle est uniquement absente de Bretagne Sud, de la région Nord-Picardie, du centre du bassin parisien et, plus ponctuellement, dans l'Est du pays (Figure 1).

Par rapport à 1995, le passage de 72 à 73 départements, interprété comme un maintien de son aire de répartition, ne doit pas cacher l'érosion généralisée de ses populations. On signale en effet des disparitions significatives de populations dans 14 départements et une baisse sensible des effectifs dans 26 départements. Dans les observations des brigades, ces évolutions sont souvent mises en relation avec des mortalités consécutives à la progression de l'écrevisse signal ( $P$. leniusculus). En fait, les effectifs ne progressent que dans les Alpes-Maritimes et la Haute-Savoie. De nouveaux signalements ponctuels de populations sont notés dans les Ardennes, la Meuse, la Moselle, les Pyrénées-Atlantiques et le Val-d'Oise. Dans le Jura, elle a disparu de $60 \%$ des sites connus en 1989.

L'espèce n'occupe que très rarement les plans d'eau, qu'ils soient publics ou privés (uniquement dans 2 et 4 départements respectivement). Elle est absente des cours d'eau de $2^{\text {ème }}$ catégorie dans la majorité des départements. En 1 ère catégorie, son taux d'occupation ne dépasse $30 \%$ du linéaire que dans 6 départements.

Elle n'est plus autant ciblée par la pêche amateur qu'en 1995. Son exploitation est non négligeable dans 34 départements (faible : 25 ; moyenne : 7 ou importante : 2) contre 40 en 1995. Aucune exploitation par la pêche professionnelle n'est signalée.

\section{L'écrevisse à pieds rouges (Astacus astacus)}

Cette espèce présente une distribution très morcelée. Elle est signalée régulièrement uniquement en Lorraine et en Bourgogne (Figure 2).

Comparée à 1995, sa distribution est stable puisque son signalement passe de 17 à 16 départements. Elle n'est plus signalée dans le Sud-Ouest (Gironde, Landes) et du Nord du pays (Seine-Maritime) et progresse en bordure et dans le Massif central (Corrèze, Loire, Puy de Dôme) où elle était signalée en 1995, ainsi que dans les Alpes (Savoie). La tendance la plus fréquente est à la disparition des populations (4 départements), bien que la situation soit souvent mal connue (6 départements). L'augmentation des effectifs ne concerne que les départements de la Loire et de la Savoie.

Contrairement aux résultats obtenus pour l'écrevisse à pieds blancs, on la trouve souvent en plans d'eau. Essentiellement dans les plans d'eau privés (9 départements) où elle fait parfois l'objet d'un élevage spécifique. En cours d'eau, elle est encore moins fréquente que l'écrevisse à pieds blancs ( $A$. pallipes) car sa présence ne dépasse jamais $30 \%$ du linéaire. Sa prédilection pour les cours d'eau de $1^{\text {ère }}$ catégorie est moins affirmée. 

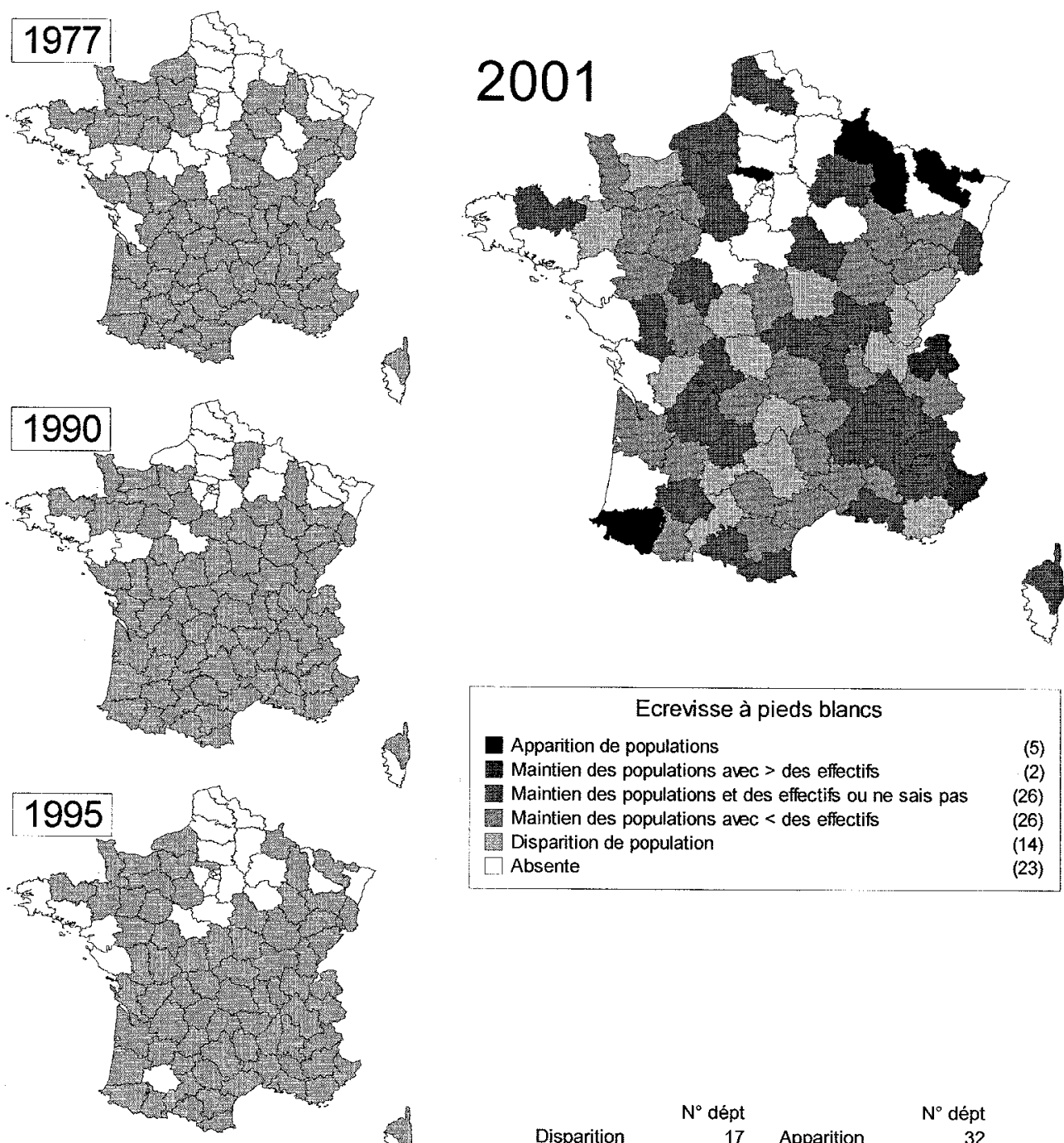

$$
\begin{array}{rrrr} 
& N^{\circ} \text { dépt } & & N^{\circ} \text { dépt } \\
\text { Disparition } & 17 & \text { Apparition } & 32 \\
& 40 & & 51 \\
& & & 62
\end{array}
$$

Source : Conseil Supérieur de la Pêche

\section{Figure 1}

Répartition de l'écrevisse à pieds blancs (Austropotamobius pallipes) en France métropolitaine par département depuis 1977. Pour les cartes des années 1977 à 1995, le signalement de l'espèce est figuré par un grisé. Pour la carte 2001, l'intensité des grisés indique la tendance inter et intra populationnelle observée à l'intérieur de chaque département conformément à la légende. Les numéros des départements où l'espèce est apparue ou a disparu par rapport à l'enquête de 1995 sont précisés au-dessus.

\section{Figure 1}

Metropolitan French distribution of Austropotamobius pallipes by department since 1977. For 1977 to 1995 maps, gray shows the presence of the species. For 2001 map, the intensity of the gray shows inter and intra population trends inside each department. The numbers identify the departments where the species has appeared or disappeared since 1995. 

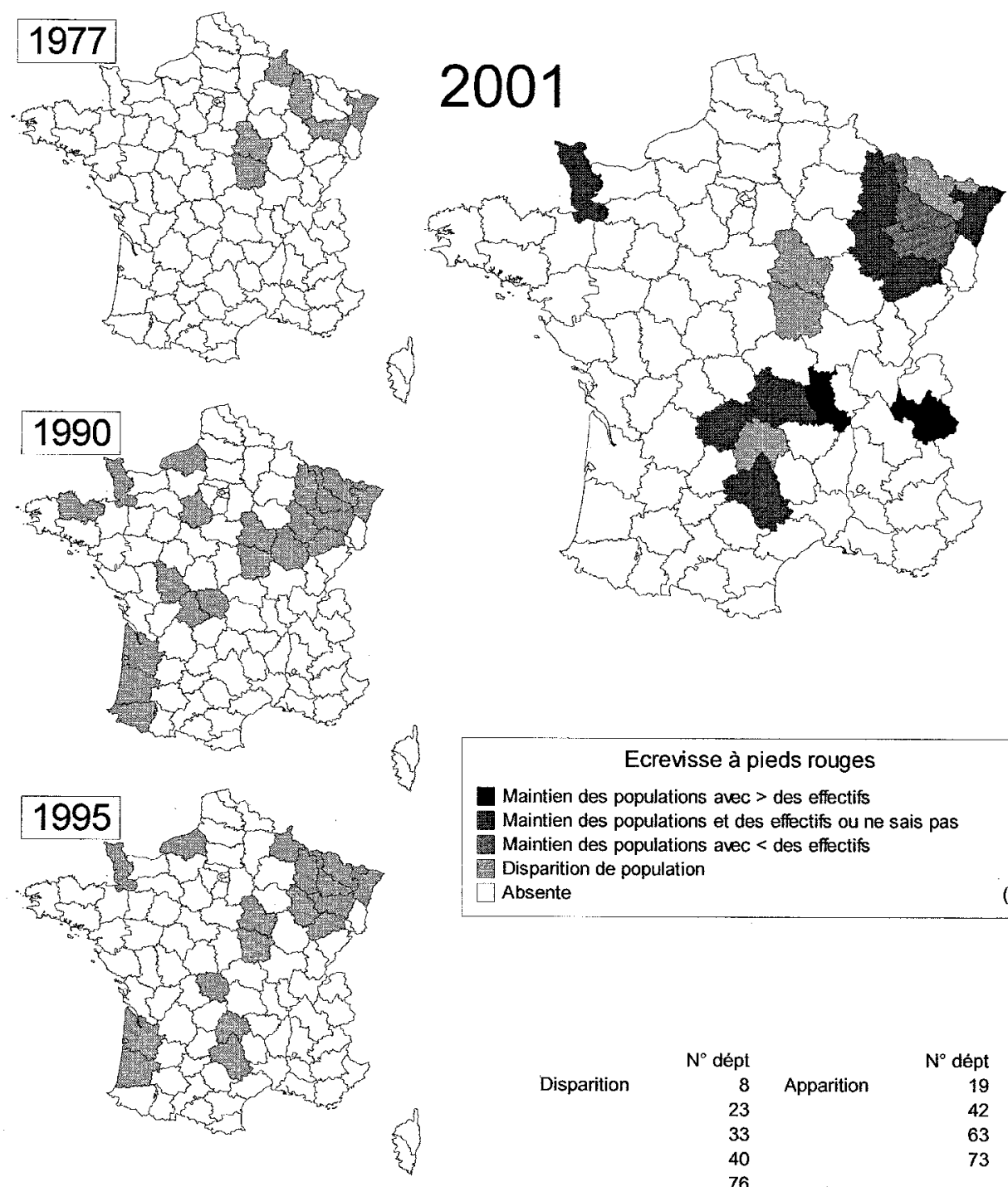

\begin{tabular}{|lr|}
\hline \multicolumn{2}{|c|}{ Ecrevisse à pieds rouges } \\
Maintien des populations avec > des effectifs & $(2)$ \\
Maintien des populations et des effectifs ou ne sais pas & $(8)$ \\
Maintien des populations avec < des effectifs & $(2)$ \\
Disparition de population & $(4)$ \\
Absente & $(80)$ \\
\hline
\end{tabular}

$\begin{array}{lrlr} & N^{\circ} \text { dépt } & N^{\circ} \text { dépt } \\ \text { Disparition } & 8 & \text { Apparition } & 19 \\ & 23 & & 42 \\ 33 & 63 \\ 40 & 73 \\ 76 & \end{array}$

\section{Figure 2}

Répartition de l'écrevisse à pieds rouges (Astacus astacus) en France métropolitaine par département depuis 1977. Légende détaillée Figure 1.

\section{Figure 2}

Metropolitan French distribution of Astacus astacus by department since 1977. Detailed legend Figure 1.

Comme pour l'écrevisse à pieds blancs, son exploitation par pêche est uniquement le fait des amateurs. En revanche, vu sa rareté, cette exploitation est cantonnée à 3 départements (Vosges, Meurthe-et-Moselle, Aveyron). 
L'écrevisse des torrents (Austropotamobius torrentium)

Cette espèce, signalée en Suisse et au Luxembourg, apparaît pour la première fois dans l'enquête CSP dans le département de la Moselle, suite à son signalement sans conteste (Figure 3). Mais l'une des deux populations connues dans ce département a déjà disparu.

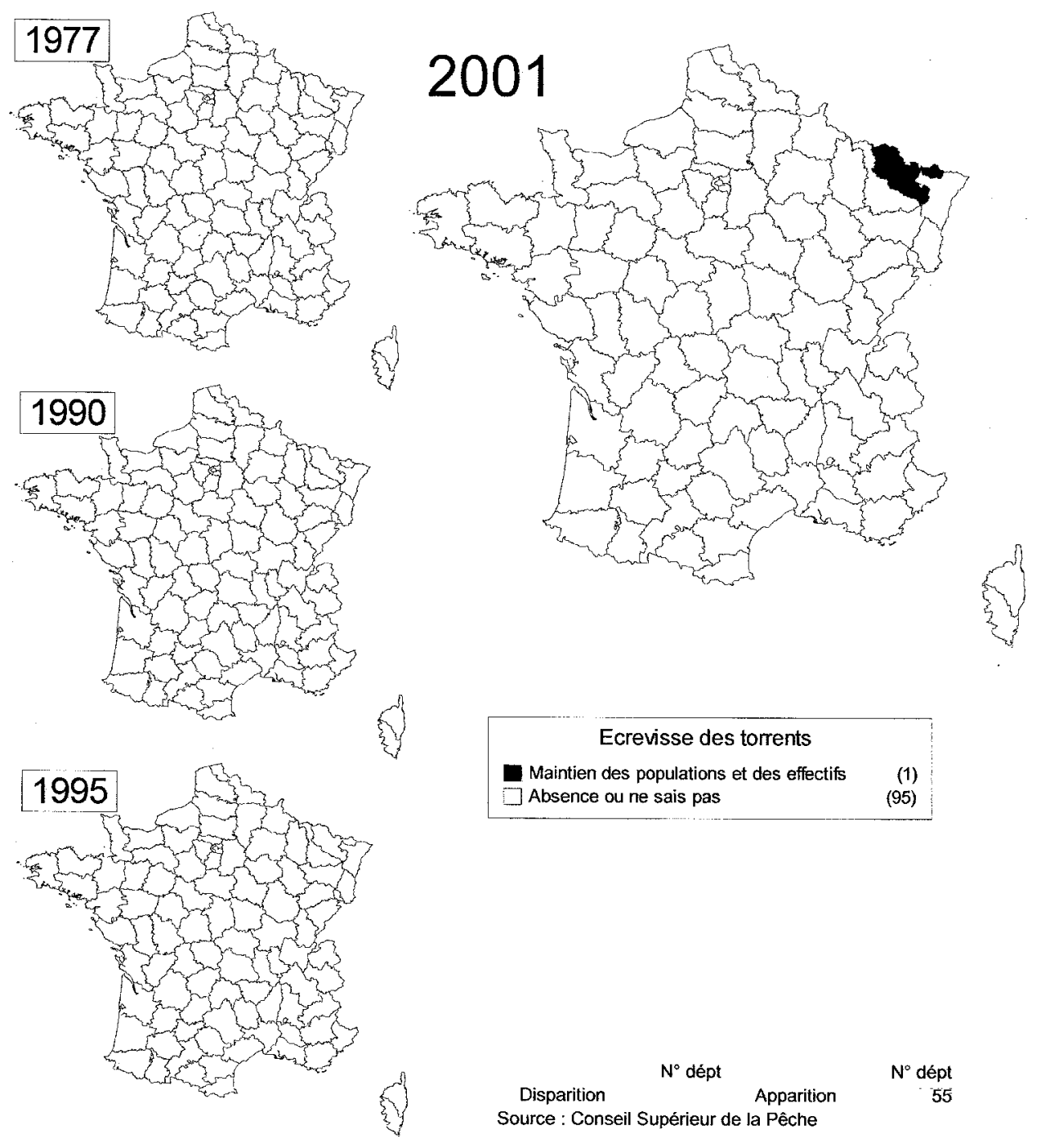

Figure 3

Répartition de l'écrevisse des torrents (Austropotamobius torrentium) en France métropolitaine par département depuis 1977. Légende détaillée Figure 1.

Figure 3

Metropolitan French distribution of Austropotamobius torrentium by department since 1977. Detailed legend Figure 1. 


\section{Espèces introduites}

\section{L'écrevisse américaine (Orconectes limosus)}

Cette espèce, présente en France depuis la fin du XIXe siècle, est la plus répandue. Elle serait encore absente uniquement du département de la Lozère (Figure 4). De 1995 à 2001, elle complète sa progression en Corse, dans le nord de la plaine orientale (HauteCorse).

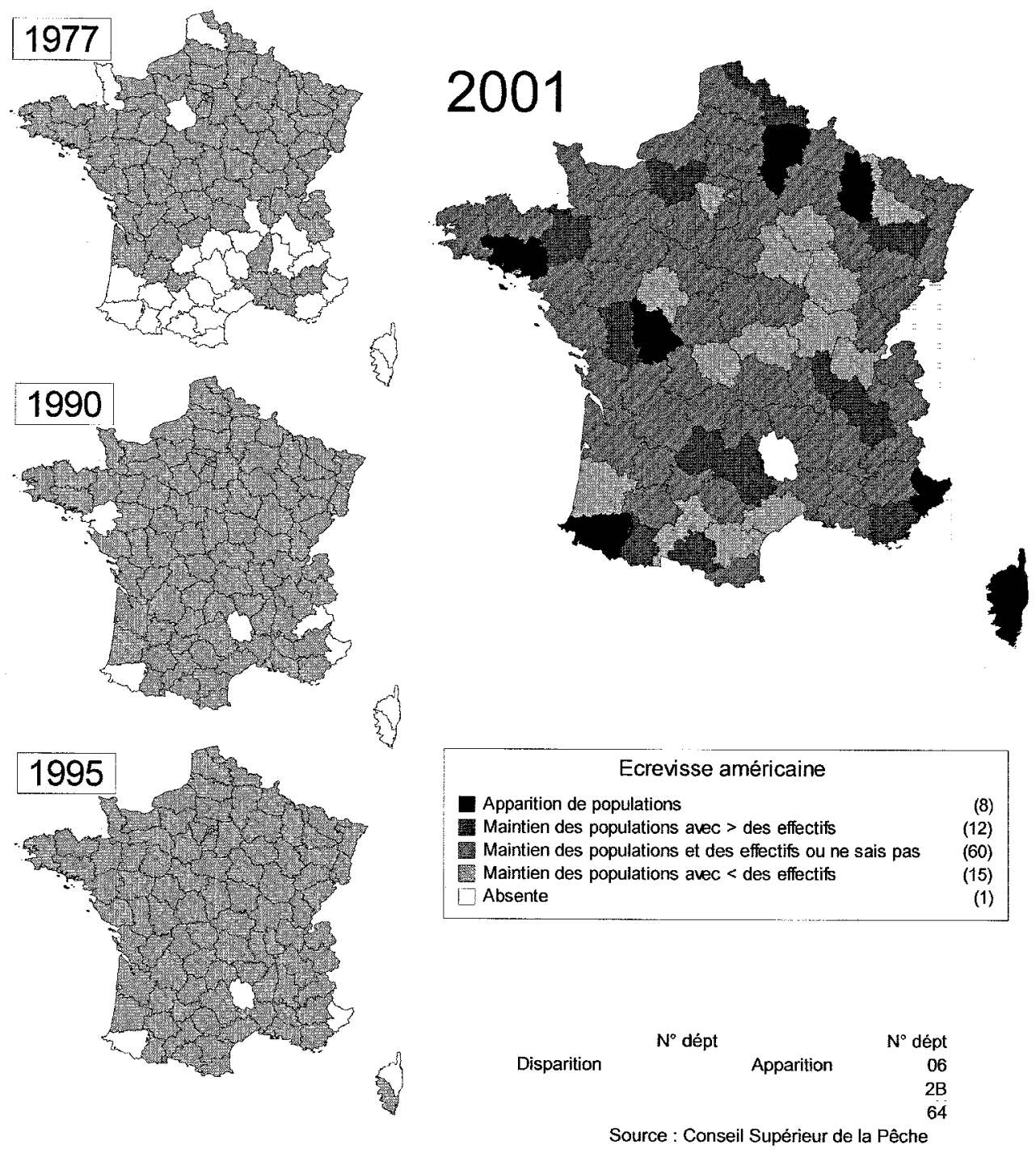

Figure 4

Répartition de l'écrevisse américaine (Orconectes limosus) en France métropolitaine par département depuis 1977. Légende détaillée Figure 1.

Figure 4

Metropolitan French distribution of Orconectes limosus by department since 1977. Detailed legend Figure 1. 
Pendant cette période, la tendance est au maintien des populations et des effectifs dans 41 départements. Tandis que les effectifs augmentent dans 12 départements avec le signalement de nouvelles populations dans 8 départements. La nouveauté est apportée par la diminution des effectifs dans 15 départements contre 4 en 1995.

Cette espèce est très abondante, en particulier dans les cours d'eau de $2^{\text {ème }}$ catégorie où elle occupe plus de $60 \%$ du linéaire dans 28 départements. Elle s'accommode également des plans d'eau, en particulier les plans d'eau publics. En marge de ces habitats, elle s'installe aussi souvent dans les eaux de $1^{\text {ère }}$ catégorie (48 départements), mais avec moins d'efficacité, puisqu'elle dépasse les $60 \%$ du linéaire uniquement dans le Loir-et-Cher, le Maine-et-Loire et la Haute-Vienne.

Dans les eaux de $2^{\text {ème }}$ catégorie, c'est actuellement l'espèce la plus recherchée, tant par les pêcheurs amateurs que par les pêcheurs professionnels. En 1999, d'importantes mortalités ont conduit les pêcheurs de la Saône aval à en abandonner l'exploitation.

\section{L'écrevisse signal (Pacifastacus leniusculus)}

En passant de 34 à 61 départements, cette espèce présente la progression la plus importante depuis 1995 (Figure 5). Même si elle n'a pas été retrouvée dans les départements du Cher, des Pyrénées-Atlantiques et des Hauts-de-Seine et reste à ce jour absente de Corse, les trois centres originels de sa progression situés dans le Limousin, le Languedoc et en Rhône-Alpes, ne sont plus perceptibles car les périmètres d'invasion se sont rejoints pour la plupart. Les discontinuités de répartition se maintiennent essentiellement en Bretagne, à la faveur de son isolement géographique, autour de l'axe Loire et sur la façade atlantique, en raison de la rareté des cours d'eau de $1^{\text {ère }}$ catégorie dans ces secteurs.

Ses populations ont tendance à se multiplier de manière encore plus sensible que l'écrevisse de Louisiane ( $P$. clarkii) puisque l'enquête en signale de nouvelles dans 30 départements (contre 14 pour cette dernière).

Elle montre une préférence pour les cours d'eau de $1^{\text {ère }}$ catégorie (présence dans les eaux de cette catégorie pour 48 départements contre 27 pour la $2^{\text {ème }}$ catégorie). Elle est signalée dans les plans d'eau publics de 9 départements ainsi que dans les plans d'eau privés de 39 départements. C'est le principal concurrent de l'écrevisse à pieds blancs $(A$. pallipes) comme le soutiennent les citations données en observation par les brigades des départements de la Creuse, de la Haute-Marne et de la Haute-Vienne.

Sa pêche peut être importante en particulier dans les rivières de 1 ère catégorie (pêche signalée dans les eaux de $1{ }^{\text {ère }}$ catégorie pour 28 départements contre 23 pour la $2^{\text {ème }}$ catégorie). C'est une cible particulière des pêcheurs amateurs dans les cours d'eau de 1 ère catégorie de l'Aveyron, du Cantal, de la Corrèze, de I'Indre, de la Loire, de la Manche, de l'Orne et de la Haute-Vienne, dans les plans d'eau publics de l'Aveyron, de la Savoie et de l'Ain ainsi que pour la pêche professionnelle dans le lac Léman et accessoirement dans les cours d'eau de $2^{\text {ème }}$ catégorie en Dordogne. Elle fait l'objet d'introduction pour la pêche, malgré l'interdiction légale (cas cité en observation dans la Vienne).

\section{L'écrevisse de Louisiane (Procambarus clarkii)}

En progressant de 33 à 49 départements cette espèce ne se répand pas autant que l'écrevisse signal car elle est moins considérée pour sa chair (Figure 6). En revanche, partout où elle s'était installée en 1995, elle s'est maintenue. 

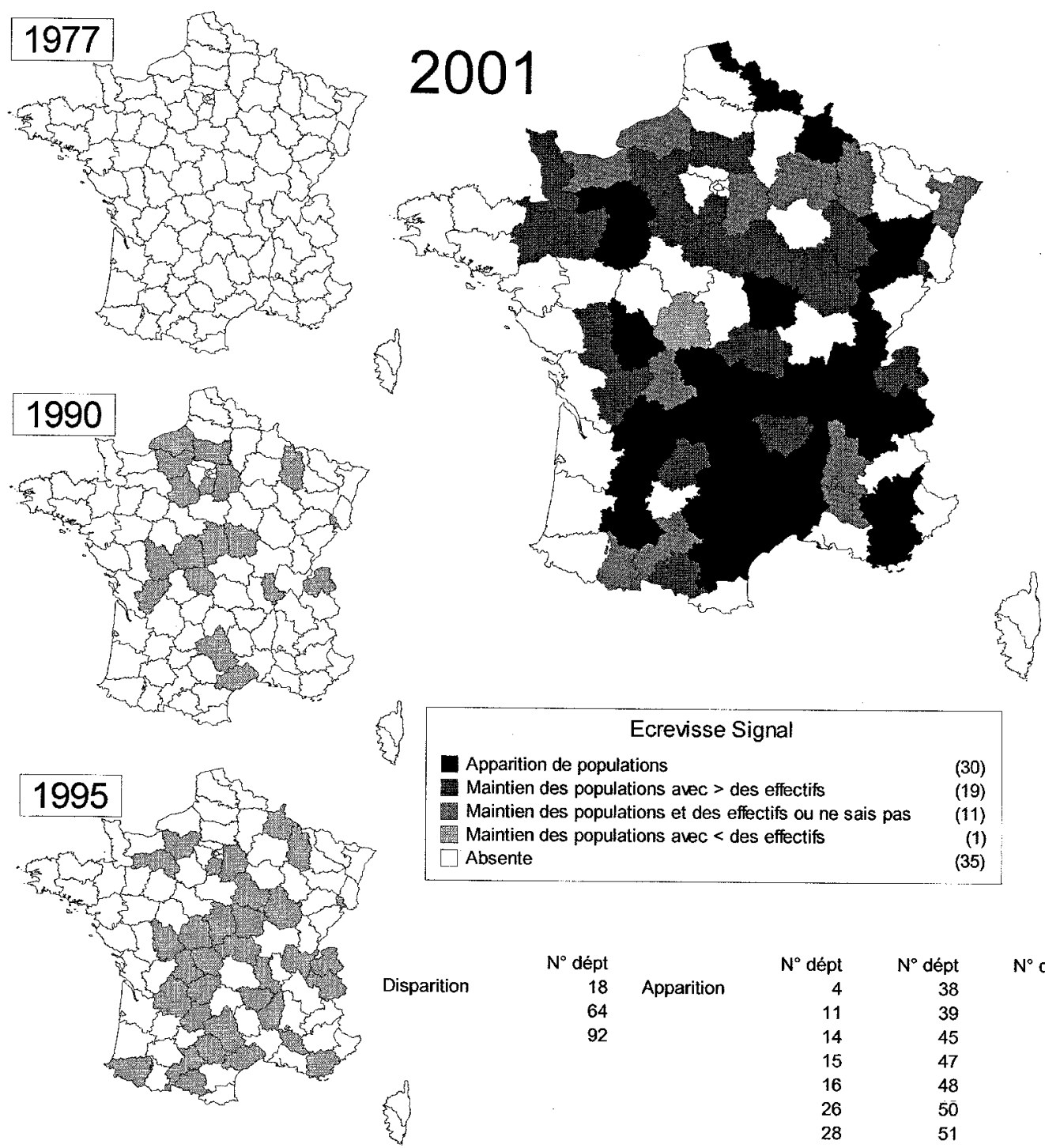

\begin{tabular}{|c|c|c|c|c|}
\hline $\mathrm{N}^{\circ}$ dépt & & $N^{\circ}$ dépt & $\mathrm{N}^{\circ}$ dépt & $\mathrm{N}^{\circ}$ dépt \\
\hline 18 & Apparition & 4 & 38 & 60 \\
\hline 64 & & 11 & 39 & 63 \\
\hline \multirow[t]{8}{*}{92} & & 14 & 45 & 65 \\
\hline & & 15 & 47 & 67 \\
\hline & & 16 & 48 & 69 \\
\hline & & 26 & 50 & 70 \\
\hline & & 28 & 51 & $\bar{i}$ \\
\hline & & 30 & 52 & 76 \\
\hline & & 32 & 53 & 79 \\
\hline & & 35 & 59 & 88 \\
\hline
\end{tabular}

\section{Figure 5}

Répartition de l'écrevisse signal (Pacifastacus leniusculus) en France métropolitaine par département depuis 1977. Légende détaillée Figure 1.

\section{Figure 5}

Metropolitan French distribution of Pacifastacus leniusculus by department since 1977. Detailed legend Figure 1.

Elle complète son occupation de la façade atlantique et du bassin de la Loire, continue sa progression vers le nord dans le bassin du Rhône, et apparaît dans le Massif central (Puy-de-Dôme) ainsi que dans le bassin du Rhin (Bas-Rhin). Vu la rapidité de sa progression dans le bassin de la Loire, le vide constitué par les départements du Maine- 


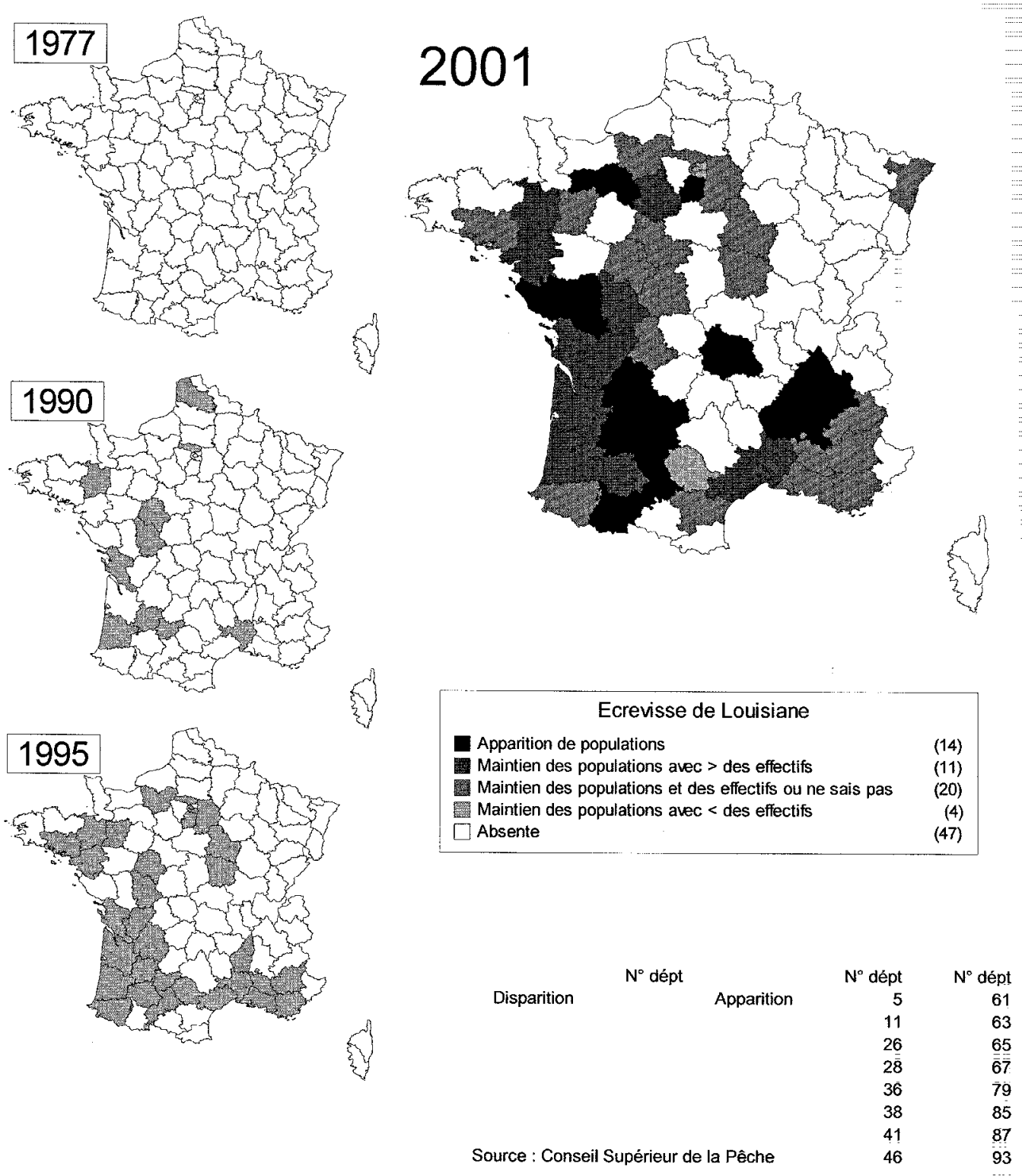

Figure 6

Répartition de l'écrevisse de Louisiane (Procambarus clarkii) en France métropolitaine par département depuis 1977. Légende détaillée Figure 1.

Figure 6

Metropolitan French distribution of Procambarus clarkii by department since 1977. Detailed legend Figure 1.

et-Loire, de la Sarthe, du Loiret et du Cher, ne devrait pas persister. Cette espèce vient de s'implanter dans le canal du Berry.

De nouvelles populations apparaissent et/ou les populations présentes augmentent leurs effectifs dans 25 départements. Les effectifs diminuent uniquement en région 
parisienne et dans le Tarn. Les départements de la Gironde, avec plus de 200 tonnes produites par an, et la Charente-Maritime sont des foyers d'infestation importants pour tout le Sud-Ouest.

Elle occupe préférentiellement les cours d'eau de $2^{\text {ème }}$ catégorie où elle dépasse les $30 \%$ du linéaire dans 10 départements. Elle est plus rare en 1 ère catégorie où elle apparaît néanmoins dans 2 départements. Sa présence en plans d'eau n'est pas négligeable ; surtout en plans d'eau privés (7 départements pour les plans d'eau publics 18 départements pour les plans d'eau privés).

Elle est exploitée par la pêche amateur dans les eaux de $2^{\text {ème }}$ catégorie de 18 départements, en particulier dans le sud (Haute-Garonne, Gers, Lot-et-Garonne, Hérault) ainsi qu'en Charente-Maritime et Loire-Atlantique. Elle est également recherchée dans les plans d'eau publics des Landes et du Vaucluse. La pêche professionnelle l'exploite accessoirement en Charente-Maritime, Dordogne et Gironde.

\section{L'écrevisse à pattes grêles (Astacus leptodactylus)}

Cette espèce fait partie des trois nouvelles espèces apparues dans les enquêtes entre 1977 et 1990. C'est actuellement la plus répandue de ces nouvelles espèces (Figure 7). Son implantation est centrée sur quatre pôles : une bande barrant l'Ouest du pays depuis la Normandie au Nord, jusqu'aux Pyrénées au Sud, l'Est du pourtour méditerranéen, la Lorraine et la Bourgogne. Elle est absente des régions les plus montagneuses (Massif Central, Rhône-Alpes) et de Corse.

Sa progression de 39 à 46 départements entre 1995 et 2001 est à mettre au crédit de nombreux nouveaux signalements, car dans le même temps l'espèce disparaît de 10 départements.

Dans la majorité des départements où elles sont représentées, les populations de cette espèce se maintiennent. Elles disparaissent en partie dans le Jura et la Vienne, alors qu'il s'en crée de nouvelles dans 9 départements, suite à son introduction dans des plans d'eau.

Contrairement aux résultats de l'enquête de 1995, cette espèce se trouve plus souvent dans les eaux de $1^{\text {ère }}$ catégorie (17 départements contre 14 pour la $2^{\text {ème }}$ ). Elle n'a pas un taux d'occupation important car elle ne dépasse jamais les $30 \%$ du linéaire. En revanche, elle est fortement représentée dans les plans d'eau, en particulier les plans d'eau privés (19 départements) où elle fait l'objet de culture en développement.

En dehors de quelques cas d'exploitation par la pêche professionnelle, dans les cours d'eau de $2^{\text {ème }}$ catégorie de la Côte d'Or et de la Dordogne, l'espèce n'est pas suffisamment abondante pour faire l'objet d'une exploitation très ciblée par pêche. En revanche, elle fait l'objet de nombreux transferts depuis les élevages vers le milieu naturel.

\section{DISCUSSION}

Les réactions obtenues suite à la publication d'une version condensée de cet article dans l'Astaciculteur de France (CHANGEUX et LAURENT, 2003), ainsi que les remarques d'un relecteur, ont permis de compléter les informations de répartition avec:

- la présence de l'écrevisse à pieds blancs ( $P$. pallipes) avérée en Meurthe-et Moselle (FRANCKHAUSER et MACHINO, 2001), suspectée dans le Bas-Rhin, ainsi que la sous-espèce italienne ( $P$. pallipes italicus) en Isère et vraisemblablement dans la Drôme (MACHINO, com. pers.) ; 

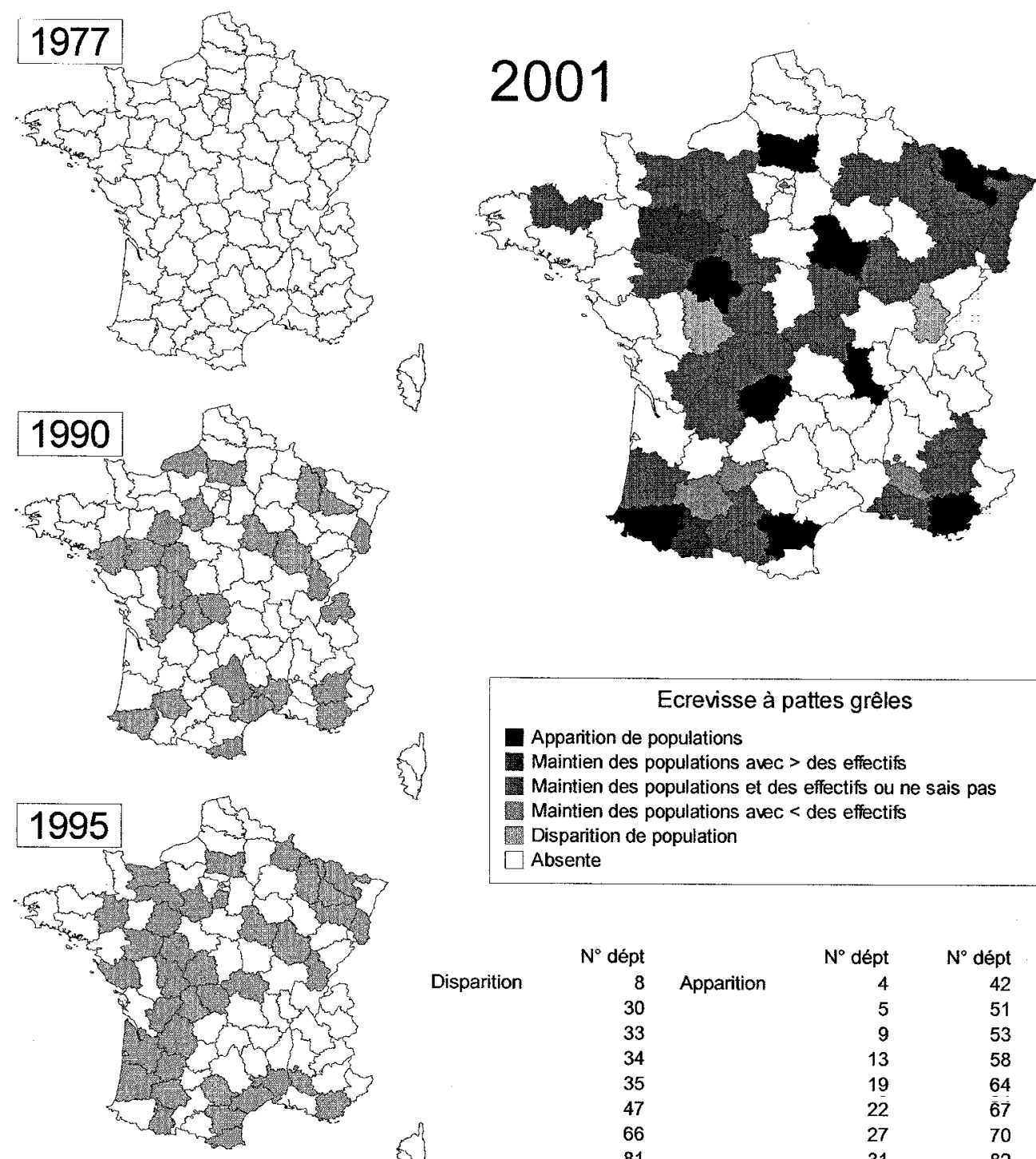

$\begin{array}{crrrr}\text { Disparition } & N^{\circ} \text { dépt } & N^{\circ} \text { dépt } & N^{\circ} \text { dépt } \\ & 8 & \text { Apparition } & 4 & 42 \\ & 30 & 5 & 51 \\ 33 & 9 & 53 \\ 34 & 13 & 58 \\ & 35 & 19 & 64 \\ 47 & 22 & \overline{6} 7 \\ & 66 & 27 & 70 \\ & 81 & 31 & 82 \\ 85 & 41 & \\ & 91 & & \end{array}$

Figure 7

Répartition de l'écrevisse à pattes grêles (Astacus leptodactylus) en France métropolitaine par département depuis 1977. Légende détaillée Figure 1.

\section{Figure 7}

Metropolitan French distribution of Astacus leptodactylus by department since 1977. Detailed legend Figure 1.

- la présence de l'écrevisse à pieds rouge (A. astacus) en Bretagne dans l'llle-etVilaine et dans les Côtes-d'Armor ;

- la présence de l'écrevisse des torrents (A. torrentium) dans le Bas-Rhin (MACHINO, com. pers.) ; 
- la présence de l'écrevisse américaine (O. limosus) dans les Landes dès 1975 et en Loire-Atlantique dès 1989 ;

- la présence de l'écrevisse signal (P. leniusculus) à Divonnes-les-Bains dans l'Ain depuis 1975 et dans la Mayenne depuis 1982 au moins ;

- la présence de l'écrevisse de Louisiane (P. clarkii) en Loire-Atlantique depuis 1984 et dans la Mayenne depuis 1985-1987;

- la présence de l'écrevisse à pattes grêles (A. leptodactylus) en Charente depuis 1990 (CASSAN, 2003) et dans les étangs privés de l'llle-et-Vilaine en 2001.

Ces ajustements, qui n'ont pas été portés sur les figures pour rester en cohérence avec la méthode, rappellent que l'absence d'une espèce n'est jamais définitive. Malgré ce défaut inhérent à tout inventaire, les enquêtes menées depuis 1977 par le CSP font très nettement ressortir le contraste entre les écrevisses autochtones qui périclitent et les écrevisses introduites qui prolifèrent. Les premières s'inscrivent à l'origine dans une zoogéographie conforme à leur histoire naturelle et à la répartition de leurs habitats, même si elles sont très marquées par les maladies importées par les écrevisses introduites. Tandis que les évolutions suivies par les écrevisses introduites sont liées de près ou de loin à l'influence humaine.

\section{Les facteurs naturels de répartition}

En France, l'écrevisse à pieds blancs, est dans le centre de son aire de répartition. Bien répandue sur le territoire, son occurrence peut encore être reliée à deux facteurs naturels principaux retenus habituellement. D'une part, au maintien de son habitat privilégié: les rivières torrentueuses au substrat grossier et aux eaux fraîches et oxygénées (LAURENT, 1988). D'autre part, à la présence de calcium, élément nécessaire à la solidification de sa carapace qu'elle renouvelle à chaque mue durant sa croissance (LOWERY, 1988). L'homme a toutefois marqué sa répartition en l'introduisant en Corse dans les années 1920. ROCHE et MATTEI (1997) signalent que l'espèce se maintient en Castagniccia (Haute-Corse) au bénéfice de son isolement géographique qui la prémunit des maladies. Comme beaucoup d'espèces autochtones occupant nos eaux douces depuis les dernières glaciations, l'écrevisse à pieds blancs présente différentes formes qui sont autant d'adaptations particulières à des conditions singulières. Certaines populations de Bretagne supportent de faibles concentrations en calcium (VIGNEUX et al., 1993). D'autres, établies en région méditerranéenne, tolèrent des températures plus élevées (FENOUIL, 1987). Enfin, les mares de la réserve du Pinail (département de la Vienne) abritent des populations génétiquement distinctes vraisemblablement attachées aux eaux stagnantes (SOUTY-GROSSET et al., 1997). De par son extension, sa diversité, et les menaces qui pèsent sur elle, c'est l'espèce d'écrevisse qui bénéficie le plus de mesures d'arrêtés de biotope (Figure 8). A elle seule, elle totalise 154 sites Natura 2000, dont 3 sont considérés comme très importants pour l'espèce.

Contrairement à l'écrevisse à pieds blancs, l'écrevisse à pieds rouges est originaire d'Europe centrale. La France se situe, avec le Portugal et l'Espagne, à l'extrémité Ouest de son aire de répartition. Elle serait autochtone dans l'Est du pays mais, depuis le moyen-âge, elle a partout fait l'objet d'introduction ce qui rend son statut difficile à établir dans l'Ouest du pays. Moins attachée à un habitat particulier que l'écrevisse à pieds blancs, elle peuple les rivières et les étangs (CUKERZIS, 1988). C'est néanmoins une espèce adaptée aux climats continentaux de son aire d'origine. La température doit descendre en dessous de $10^{\circ} \mathrm{C}$ pour que la reproduction se déclenche et son optimum de croissance se situe entre 18 et $22^{\circ} \mathrm{C}$. Elle présente une bonne aptitude 


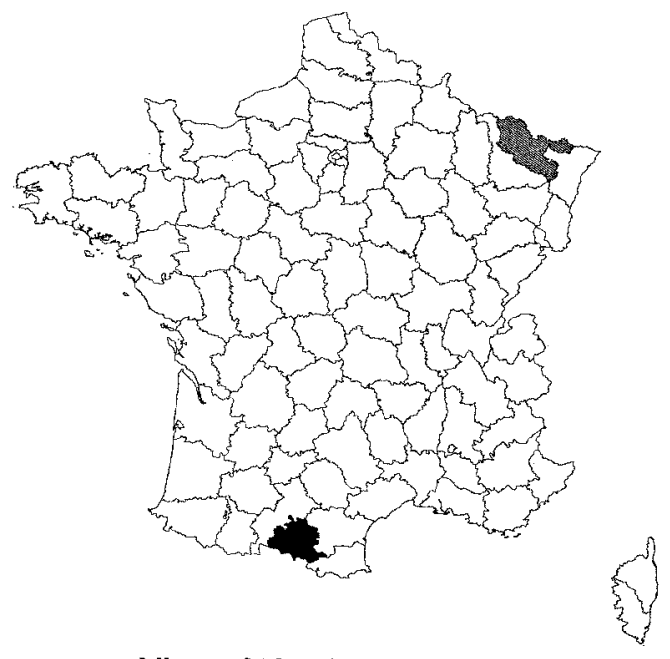

$\mathrm{Nb}$ arrêtés de biotope Ecrevisse à pieds rouges

5 (1)

2 (1)

0 (94)

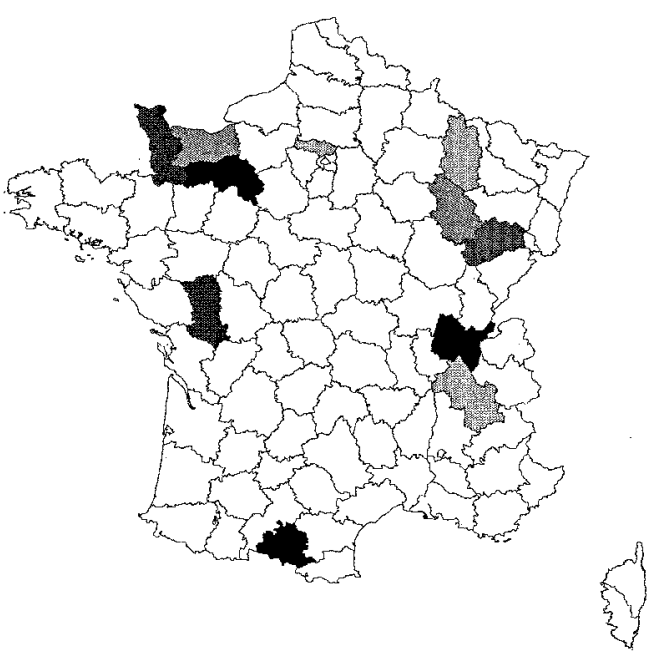

$\mathrm{Nb}$ arrêtés de biotope Ecrevisse à pieds blancs

\begin{tabular}{|c|c|}
\hline od & \\
\hline 4 à & (2) \\
\hline 酾 3 à & (1) \\
\hline 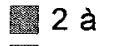 & (2) \\
\hline 1 à & (3) \\
\hline & \\
\hline
\end{tabular}

Figure 8

Effectifs départementaux des arrêtés de biotopes (source : Service du Patrimoine Naturel du Muséum National d'Histoire Naturelle).

Figure 8

Number of French habitat decrees by department (source : Service du Patrimoine Naturel du Muséum National d'Histoire Naturelle).

à l'élevage en étang d'eau froide mais l'enquête montre qu'elle n'occupe pas tous les habitats disponibles. Le morcellement de sa distribution est plutôt lié au hasard des repeuplements. Le succès à long terme de ces opérations est moins évident car l'espèce est globalement en régression ; vraisemblablement en raison de sa sensibilité vis-à-vis de la peste de l'écrevisse. Moins répandue que l'écrevisse à pieds blancs, le nombre d'arrêtés de biotopes pris en faveur de l'écrevisse à pieds rouges est de très loin inférieur (7 contre 48 en faveur de l'écrevisse à pieds blancs). II est à noter que 5 arrêtés pris en faveur de l'écrevisse à pieds rouges sont situés en Ariège où sa présence n'est pas signalée dans l'enquête.

\section{L'influence humaine}

L'activité humaine qui fait courir le plus de risques aux populations d'écrevisses peut sembler anodine pour le grand public : c'est la dissémination pour la pêche et l'élevage. En effet, les introductions successives des espèces américaines ont répandu en plusieurs vagues la peste de l'écrevisse (MACHINO et DIEGUEZ-URIBEONDO, 1998). L'écrevisse 
signal et l'écrevisse de Louisiane sont des réservoirs permanents de cette maladie (NEVEU, 1998a; NEVEU, 2000). Lors de la dernière enquête CSP, menée en 1995, il semblait qu'elle n'était plus aussi fréquente. On signalait alors une autre pathologie dans le pays basque espagnol : la maladie de la porcelaine (Thelohania contejeani), dont l'évolution est plus lente et moins visible (DIEGUEZ-URIBEONDO et al., 1997). Mais le retour de la peste a été signalé sans ambiguïté dès 1990 dans l'Ouest de la France (NEVEU, 1998b) et plus récemment dans les départements des Vosges (COLLAS et SALEK, 2002), de la Marne (MACHINO et DIEGUEZ-URIBEONDO, 1998), et des Deux-Sèvres (NEVEU et BACHELIER, 2003). Un virus a également été identifié comme responsable d'une mortalité particulière en Ardèche (EDGERTON et al., 2002).

\section{Tableau I}

Comparaison des paramètres démographiques des principales espèces d'écrevisses (modifié selon HOLDICH et LOWERY, 1988). avec ASA : écrevisse à pieds rouges, APP : écrevisse à pieds blancs, ASL : écrevisse à pattes grêles, OCL : écrevisse américaine, PFL : écrevisse signal, PCC : écrevisse de Louisiane. La stratégie r-K fait référence à la classification de PIANKA, 1970.

\section{Table I}

Comparison between demographic parameters of the principal crayfish species (modified from HOLDICH and LOWERY, 1988), with ASA : Astacus astacus, APP : Austropotamobius pallipes, ASL: Astacus leptodactylus, OCL: Orconectes limosus, PFL: Pacifastacus leniusculus, PCC: Procambarus clarkii. The r-K strategy refers to PIANKA (1970)'s classification.

\begin{tabular}{lccc} 
& APP & ASA & ASL \\
\hline Longévité (année) & +11 maxi. & $10-15$ maxi. & $6-9$ \\
Age à maturité (nb d'étés) & $3-4$ & $2-4$ & $2-3$ \\
Longueur totale à maturité $(\mathrm{mm})$ & $88-60$ & $70-80$ & $75-82$ \\
Croissance & lente & Modérée & rapide \\
Nb d'œufs & 130 maxi. & $100-150$ & $200-400$ \\
Possibilités de reprod./an & 1 & 1 & 1 \\
\hline Stratégie & $\mathbf{K}$ & $\mathbf{K}$ & $\mathbf{K}-\mathbf{r}$ \\
\hline & $\mathbf{P F L}$ & $\mathbf{P C C}$ & $\mathbf{O C L}$ \\
\hline Longévité (année) & 9 maxi. & 2 & 4 \\
Age à maturité (nb d'étés) & $2-3$ & 1 & $1-2$ \\
Longueur totale à maturité $(\mathrm{mm})$ & $80-90$ & $60-90$ & $50-40$ \\
Croissance & modérée & très rapide & modérée \\
Nb d'œufs & $110-300$ & $50-600$ & $100-200$ \\
Possibilités de reprod./an & 1 & plusieurs & plusieurs \\
\hline Stratégie & $\mathbf{K}-\mathbf{r}$ & $\mathbf{r}$ & $\mathbf{r}$ \\
\hline
\end{tabular}


L'introduction d'espèces américaine n'est pas nécessaire pour que la peste se déclare. Les spores d'Aphanomyces astaci sont également disséminés lors des introductions de poissons issus de secteurs contaminés (OIDTMANN et al., 2002) ou bien même simplement par les pattes des oiseaux aquatiques ainsi que par les bottes des pêcheurs. C'est de cette manière que les populations d'écrevisses irlandaises, pourtant protégées par leur insularité, ont été menacées par la peste (REYNOLDS, 1988).

Les disparitions de populations d'écrevisses à pieds blancs sont parfois compensées par la détection de nouvelles populations. On peut relier cela à une intensification des prospections. Toutefois, un fonctionnement cyclique des populations, où la survie de quelques individus dans un habitat resté favorable permet à une population de se reconstituer après une importante mortalité, est signalée dans l'enquête par les brigades du Jura et de l'Ardèche.

Au-delà des épizooties, les espèces introduites présentent généralement des caractéristiques démographiques qui les avantagent en situation de compétition (Tableau I). Elles peuvent se reproduire plus jeunes. Elles sont plus prolifiques et croissent plus rapidement.

L'enquête met en évidence des signes de faiblesse pour certaines populations d'écrevisses américaines (O. limosus), installées maintenant depuis plus d'un siècle. C'est un processus habituel de la progression d'une espèce introduite, qui commence par occuper tout l'espace favorable, puis voit ses effectifs régulés par l'apparition de maladies ou de compétitions qui n'existaient pas lorsqu'elle était en situation pionnière.

Ce n'est pas le cas des deux autres espèces américaines ( $P$. leniusculus et $P$. clarkii), qui sont encore en phase de colonisation. D'autant plus qu'il existe un véritable engouement pour ces deux espèces peu exigeantes qui présentent en outre de bonnes qualités organoleptiques. L'écrevisse signal, qui peut occuper les étangs comme les rivières, concurrence directement l'écrevisse à pieds rouges comme l'écrevisse à pieds blancs. L'écrevisse de Louisiane, en revanche, ne menace pas aussi directement les espèces autochtones car elle occupe plus particulièrement les marécages où ne subsiste plus que l'écrevisse américaine (O. limosus). En revanche, de par son adaptation aux zones humides temporaires dont elle est originaire, lorsque le marais s'assèche, elle s'enterre dans des galeries profondes. Cela peut endommager gravement les digues (HUNER, 1988). En outre, elle peut se déplacer hors de l'eau pour coloniser des milieux favorables (BUILLES et al., 2000).

Outre le risque sanitaire, l'élevage d'écrevisses à pieds rouges et à pieds blancs présente généralement des rendements faibles, aussi les élevages de ce type sont très peu nombreux ce qui limite les possibilités de repeuplement. L'écrevisse à pattes grêles a été importée de Turquie pour pallier cet inconvénient. De par ses meilleures qualités démographiques (Tableau I), elle présente une meilleure aptitude à l'élevage sans porter préjudice aux espèces autochtones car elle n'est pas porteuse saine de la peste. Elle serait également plus résistante que les autres espèces sensibles à ces maladies. De fait, aucun cas de concurrence avec une population d'écrevisses à pieds rouges ou d'écrevisses à pieds blancs n'est signalé dans l'enquête.

\section{CONCLUSION}

La forme adoptée par l'enquête nationale ne rentre pas dans le détail de toutes les informations détenues par les agents du CSP. La précision est néanmoins suffisante pour démontrer que les espèces autochtones sont soit rares, soit en forte régression, 
alors que les espèces introduites progressent malgré toutes les mesures prises contre leur dissémination. L'interdiction d'introduction et de transport vivant n'ont pas empêché l'accélération de la progression de l'écrevisse signal ces cinq dernières années, à l'origine de la recrudescence des cas de peste dans les zones de contact. On évalue ici, sur le moyen terme, la limite de mesures basées sur une liste positive (espèces présentes et admises à l'introduction) et une liste négative (espèces susceptibles de provoquer des déséquilibres biologiques, interdites à l'introduction et au transport vivant) dont l'application est étendue à tout le territoire d'un pays et qui n'ont jamais fait l'objet de sanction sérieuse à l'encontre de contrevenant. S'il n'est pas question de revenir sur ce type de mesures, par ailleurs préconisées dans d'autres pays (LODGE et al., 2000), leur efficacité gagnerait à être relayée par une campagne de sensibilisation auprès du grand public mettant en avant le danger que représentent l'écrevisse signal et l'écrevisse de Louisiane pour les espèces autochtones.

Au même titre que les parcours «no kill » ou l'augmentation des tailles légales retenues pour les poissons, les mesures de protection par la limitation des prélèvements par pêche sont de plus en plus mises en œuvre par les pêcheurs eux-même car ils sont prêts à faire des sacrifices pour préserver les écrevisses autochtones. De même, des programmes de réintroduction sont développés à partir d'élevages pour le repeuplement. Malheureusement, tous ces efforts ne semblent pas inverser la tendance.

Il semble donc nécessaire de mettre en place une nouvelle politique de conservation, centrée sur des espaces plus restreints et mettant l'accent sur la préservation de l'habitat et le respect de certaines règles de prophylaxie à déterminer (contrôle des introductions d'écrevisses et de poissons, désinfection des bottes, etc.). Adopter un dispositif intermédiaire, tel que celui mis en place au Royaume-Uni en 1996, avec d'importantes surfaces de l'Angleterre et du Pays de Galles désignées en «no-go-areas " (HOLDICH et al., 1999), ne peut s'envisager qu'en substitution de l'actuel dispositif et risque de présenter les mêmes difficultés d'application et de contrôle. La solution la plus novatrice et complémentaire semble être de délimiter de petits périmètres facilement contrôlables et respectant le découpage en bassins hydrographiques. Cela ne peut pas s'envisager sans un fort engagement associatif et administratif local. Dans l'arsenal réglementaire actuel, l'arrêté de biotope et son plan de gestion sont une voie possible mais limitée alors que le nombre important de sites référencés Natura 2000 pour l'écrevisse à pieds blancs (4 ${ }^{\mathrm{ème}}$ espèce d'invertébré la plus signalée) permet d'envisager la conservation de l'espèce en proportion avec les menaces qui pèsent sur elle. Le cas de l'île Corse, récemment colonisée par l'écrevisse américaine (O. limosus), mériterait de faire l'objet de mesures particulières pour éradiquer cette dernière et pour éviter que l'écrevisse signal et l'écrevisse de Louisiane ne la suivent dans les années qui viennent.

Ces démarches plus localisées, ou prenant en compte les situations insulaires, semblent plus à même de faire face à l'évolution prévisible de la situation dans les années à venir où l'essentiel des bassins susceptibles de les accueillir seront occupés par des écrevisses introduites et où les espèces autochtones, qui sont déjà en danger, ne survivront que dans des espaces limités et protégés (TAUGBØL et SKURDAL, 1999).

Enfin, pour statuer plus précisément sur les variations d'abondance et d'occurrence (afin par exemple de mieux connaître le fonctionnement des populations et contribuer à établir une stratégie de conservation), il serait nécessaire de définir un protocole commun aux différents atlas et recensements réalisés localement par les agents du CSP. Ce protocole, qu'il est prévu d'intégrer dans le Réseau d'Observation des Milieux (ROM) développé par l'établissement autour du découpage en contextes (voir programme 4 inscrit au contrat d'objectif dans ANONYME, 2001), devra notamment s'attacher à mesurer l'intensité de prospection pour pondérer les résultats. 


\section{REMERCIEMENTS}

Je remercie J.-P. AUXIĖTRE, M. BRAMARD et un relecteur anonyme pour les remarques constructives faites sur ce texte, ainsi que l'ensemble des personnels du CSP qui a participé à cette enquête et aux précédentes.

\section{BIBLIOGRAPHIE}

ANONYME, 2001. Le contrat d'objectif. Numéro spécial Eaux libres, 29, 20-22.

ARRIGNON J., 1990. L'enquête nationale sur les écrevisses: une réglementation inadaptée. Eaux libres, 2, 3-8.

BUILLES S., TERRIER O., VIGNAUD M., 2000. Étude préliminaire sur l'écrevisse Procambarus clarkii, Réserve naturelle des marais de Bruges (Gironde). Rapport SEPANSO, Conseil Supérieur de la Pêche, Réserves Naturelles de France, $49 \mathrm{p}$.

CASSAN Y., 2003. Astacus leptodactylus: des raisons d'espérer. L'Astaciculteur de France, 77, 17.

CHANGEUX T., 1996. Premiers résultats de l'enquête express écrevisses. Conseil Supérieur de la Pêche, Circulaire D003 du 15/01/95, 5 p.

CHANGEUX T., LAURENT P.J., 2003. Répartition et évolution des populations d'écrevisses en France telle que le font apparaître les résultats de l'enquête menée par le CSP (Conseil supérieur de la pêche) en 2001. L'Astaciculteur de France, 76, 5-14.

COLLAS M., SALEK X., 2002. Description d'un cas de peste de l'écrevisse ou aphanomycose dans le département des Vosges. L'Astaciculteur de France, 70, 2-6.

CUKERZIS J.M., 1988. Astacus astacus in Europe. In : HOLDICH D.M., LOWERY R.S. (Eds.), Freshwater crayfish: biology, management and exploitation, The University Press, Cambridge, 309-340.

DIEGUEZ-URIBEONDO J., PINEDO-RUIZ J., MUZQUIZ J.L.1997., Thelohania contejeani in the province of Alava, Spain. Bull. Fr. Pêche Piscic., 347, 749-752.

EDGERTON B.F., WATT H., BACHERAS J.-M., BONAMI J.-R., 2002. An intranuclear bacilliform virus associated with near extirpation of Austropotamobius pallipes from the Nant watershed in Ardèche, France. Journal of Fish Diseases, 25, 523-531.

FENOUIL E., 1987. Biologie et développement de l'écrevisse Austropotamobius pallipes en région provençale. Thèse Univ. Aix-Marseille, $290 \mathrm{p}$.

FRANCKHAUSER R., MACHINO Y., 2001. L'écrevisse de torrents (Austropotamobius torrentium) en France. L'Astaciculteur de France, 68, 2-5.

HOLDICH D.M., LOWERY R.S. (Eds.), Freshwater crayfish: biology, management and exploitation, The University Press, Cambridge, 408 p.

HOLDICH D.M., ROGERS W.D., REYNOLDS J.D., 1999. Crayfish in the british isles. In: GHERARDI F., HOLDICH D.M. (Eds.), Crayfish in Europe as alien species. How to make the best of a bad situation ? Crustacean issues 11, A.A. Balkema, Rotterdam and Brookfield, 221-235. 
HUNER J.V., 1988. Procambarus in North America and elsewhere. In: HOLDICH D.M., LOWERY R.S. (Eds.), Freshwater crayfish: biology, management and exploitation, The University Press, Cambridge, 239-261.

LAURENT P.J., 1988. Austropotamobius pallipes and A. torrentium with observations on their interaction with other species in Europe. In: HOLDICH D.M., LOWERY R.S. (Eds.), Freshwater crayfish : biology, management and exploitation, The University Press, Cambridge, 341-364.

LODGE D., TAYLOR C., HOLDICH D., SKURDAL J., 2000. Reducing impacts of exotic crayfish introduction: new policies needed. Fisheries, 25(8), 21-23.

LOWERY R.S., 1988. Growth, moulting and reproduction. In: HOLDICH D.M., LOWERY R.S. (Eds.), Freshwater crayfish: biology, management and exploitation, The University Press, Cambridge, 83-113.

MACHINO Y., 1995. Austropotamobius torrentium (Schrank, 1803) existe bel et bien en France. L'Astaciculteur de France, 45, 17-19.

MACHINO Y., 1996. Nouveau site de l'écrevisse des torrents (Austropotamobius torrentium Schrank, 1803) en France. L'Astaciculteur de France, 48, 2-3.

MACHINO Y., DIEGUEZ-URIBEONDO J., 1998. Un cas de peste de l'écrevisse en France dans le bassin de la Seine. L'Astacicuteur de France, 54, 2-11.

NEVEU A., 1998a. Pacifastacus leniusculus : son rôle de vecteur et de réservoir de la peste des écrevisses (aphanomycose), état actuel des connaissances. L'Astaciculteur de France, 57, 6-11.

NEVEU A., 1998b. Présence de l'aphanomycose en France : suivi d'un foyer dans l'Ouest de 1990 à 1998. L'Astaciculteur de France, 57, 2-5.

NEVEU A., 2000. L'écrevisse de Louisiane (Procambarus clarkii) : réservoir permanent et vecteur saisonnier de l'aphanomycose dans un étang de l'Ouest de la France. L'Astaciculteur de France, 63, 7-11.

NEVEU A., BACHELIER E., 2003. Mortalité d'Austropotamobius pallipes sur le bassin de la Sèvre Niortaise. Présence de l'aphanomycose. L'Astaciculteur de France, 76, 2-4.

OIDTMANN B., HEITZ D., RODGERS D., HOFFMANN R.W., 2002. Transmission of crayfish plague. Diseases of Aquatic Organisms, 52, 159-167.

PIANKA, E.R., 1970. On r- and K-selection. American naturalist, 104, 592-597.

REYNOLDS J.D., 1988. Crayfish extinctions and crayfish plague in central Ireland. Biological Conservation, 45, 279-285.

ROCHE B., MATTEI J., 1997. Les espèces animales introduites dans les eaux douces de Corse. Bull. Fr. Pêche Piscic., 344/345, 233-239.

SOUTY-GROSSET C., GRANDJEAN F., RAIMOND R., FRELON M., DEBENEST C., BRAMARD M., 1997. Conservation genetics of white-clawed crayfish Austropotamobius pallipes: the usefulness of the mitochondrial DNA marker. Bull. Fr. Pêche Piscic., 347 : 677-692.

TAUGBØL T., SKURDAL J., 1999. The future of native crayfish in Europe. In: GHERARDI F., HOLDICH D.M. (Eds.), Crayfish in Europe as alien species. How to make the best of a bad situation ? Crustacean issues 11, A.A. Balkema, Rotterdam and Brookfield, 271-279. 
VIGNEUX E., THIBAULT M., MARNELL F., SOUTY-GROSSET C., 2002. National legislation, EU directives and conservation. Bull. Fr. Pêche Piscic., 367, 887-898.

VIGNEUX E., KEITH P., NOËL P., 1993. Atlas préliminaire des crustacés décapodes d'eau douce de France. Coll. Patrimoines Naturels, Vol. 14, SFF, BIMM-LNHN, CSP, Min. Env., Paris, $55 \mathrm{p}$. 


\section{Annexe 1 \\ Questionnaire utilisé dans l'enquête.}

\section{Annex 1}

Questionnaire form used in the survey.

\section{NOTES EXPLICATIVES \\ POUR L'ENQUETE EXPRESS ÉCREVISSE}

Pour alléger le questionnaire

les informations à fournir concernent le département dans son ensemble

(1) Code espèce

ASA : Astacus astacus, écrevisse à pattes ou à pieds rouges

APP : Austropotamobius pallipes, écrevisse à pieds blancs

APT : Austropotamobius torrentium, écrevisse des torrents

ASL : Astacus leptodactylus, écrevisse à pattes grêles ou turque

OCL : Orconectes limosus, écrevisse américaine

PFL : Pacifastacus leniusculus, écrevisse de Californie

PCC : Procambarus clarkii, écrevisse de Louisianne

Autres espèces d'éctevisses à préciser par un code indiqué dans

"observations générales"

(2) Tendance majeure dans le département depuis l'inventaire de 1995 (résultats exposés dans la circulaire D003 du 15 janvier 1996)

-- : Disparition de populations

- Maintien des populations avec diminution des effectifs

0 : Maintien des populations et des effectifs

+ : Maintien des populations avec augmentation des effectifs

++ : Apparition de populations

NSP : Ne sais pas

(3) Indice d'abondance dans le département

0 : Absente dans les cours d'eau de la catégorie concernée

+ : Présence faible (comprise entre 1 \% et 30 \% du linéaire)

++ : Présence moyenne (comprise entre $30 \%$ et $60 \%$ du linéaire)

+++ : Présence importante (comprise entre 60 o et 100 o du linéaire)

NSP : Ne sais pas

(4) Exploitation par pêche dans le département

I : Pêche interdite

0 : Pas d'exploitation (sans intérêt halieutique)

+ : Exploitation faible (capture accessoire)

++ : Exploitation moyenne (espèce cible)

+++ : Exploitation importante (espèce très recherchée)

NSP : Ne sais pas

Remarque : Les productions peuvent être faibles malgré une exploitation importante si les stocks sont petits. 


\section{FICHE D'ENQUETE EXPRESS ECREVISSE}

à renvoyer avant le 15 septembre 2001

au

Service Technique

Direction générale du CSE

134, avenue de Malakoff

75116 PARIS

Sur l'ensemble du département de $: \ldots \ldots \ldots \ldots$

\begin{tabular}{|l|l|l|}
\hline $\begin{array}{l}\text { Espèce } \\
(1)\end{array}$ & $\begin{array}{l}\text { Présence } \\
\text { (oui/non) }\end{array}$ & $\begin{array}{l}\text { Tendance } \\
\text { majeure (2) }\end{array}$ \\
\hline ASA & & \\
\hline APP & & \\
\hline AST & & \\
\hline OCL & & \\
\hline PEL & & \\
\hline PCC & & \\
\hline APT & & \\
\hline & & \\
\hline
\end{tabular}

Cours d'eau de lère catégorie

\begin{tabular}{|l|l|l|}
\hline $\begin{array}{l}\text { Espèce } \\
\text { (1) }\end{array}$ & $\begin{array}{l}\text { Ind. (3) } \\
\text { d'abon. }\end{array}$ & $\begin{array}{l}\text { Exploit. (4) } \\
\text { pêche amat. }\end{array}$ \\
\hline ASA & & \\
\hline ARP & & \\
\hline ASL & & \\
\hline OCL & & \\
\hline PEL & & \\
\hline PCC & & \\
\hline APT & & \\
\hline & & \\
\hline
\end{tabular}

Cours d'eau de 2 de catégorie

\begin{tabular}{|l|l|l|l|}
\hline $\begin{array}{l}\text { Espèce } \\
(1)\end{array}$ & $\begin{array}{l}\text { Ind. (3) } \\
\text { d'abon. }\end{array}$ & $\begin{array}{l}\text { Exploit. } \\
(4) \\
\text { pêche } \\
\text { amat. }\end{array}$ & $\begin{array}{l}\text { Exploit. } \\
\text { (4) } \\
\text { pêche } \\
\text { pro. }\end{array}$ \\
\hline ASA & & & \\
\hline APP & & & \\
\hline ASL & & & \\
\hline OCL & & & \\
\hline PFL & & & \\
\hline PCC & & & \\
\hline APT & & & \\
\hline & & & \\
\hline
\end{tabular}

T.S.V.P

* Consulter les notes explicatives jointes pour remplir les Tableaux 
Plans d'eau publics (domaniaux ou communaux)

\begin{tabular}{|l|l|l|l|}
\hline $\begin{array}{l}\text { Espèce } \\
(1)\end{array}$ & $\begin{array}{l}\text { Nb de } \\
\text { sites }\end{array}$ & $\begin{array}{l}\text { Exploit. (4) } \\
\text { pêche amat. }\end{array}$ & $\begin{array}{l}\text { Exploit. } \\
\text { (4) } \\
\text { pêche } \\
\text { pro. }\end{array}$ \\
\hline ASA & & & \\
\hline APP & & & \\
\hline ASL & & & \\
\hline OCL & & & \\
\hline PEL & & & \\
\hline PCC & & & \\
\hline APT & & & \\
\hline
\end{tabular}

Nb total approximatif de plans d'eau publics dans le département :......
Plans d'eau privés ou unités d'exploitation

\begin{tabular}{|l|l|l|}
\hline $\begin{array}{l}\text { Espèce } \\
(1)\end{array}$ & $\begin{array}{l}\text { Nb de } \\
\text { sites }\end{array}$ & $\begin{array}{l}\text { Production (en kg) } \\
\text { annuelle estimée }\end{array}$ \\
\hline ASA & & \\
\hline APP & & \\
\hline ASL & & \\
\hline OCL & & \\
\hline PEL & & \\
\hline PCC & & \\
\hline APT & & \\
\hline & & \\
\hline
\end{tabular}

$\mathrm{Nb}$ total approximatif de plans d'eau privés ou d'unités d'exploitation dans le département :......

\section{OBSERVATIONS GENERALES}

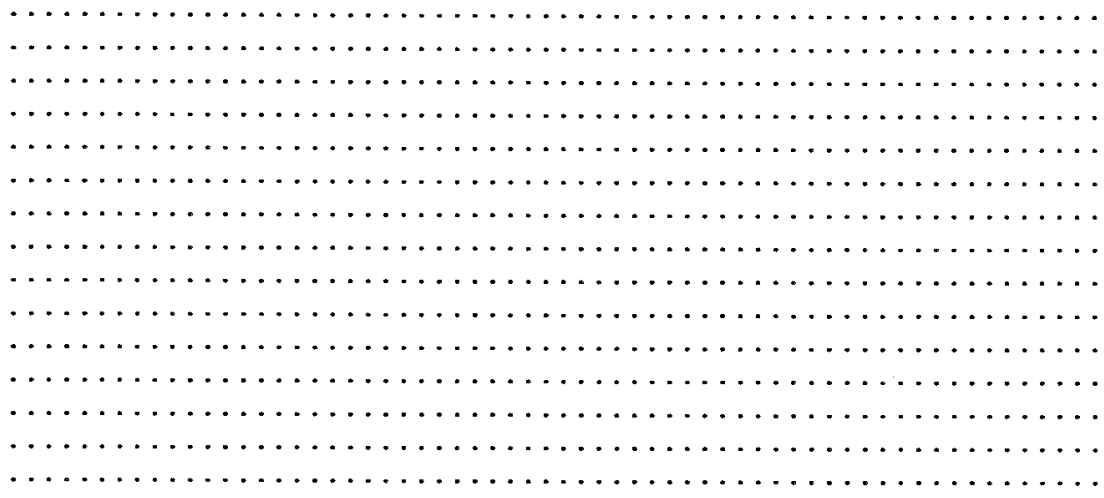

Fait à 


\section{Annexe 2}

Tableau de contingence des réponses en nombre de départements (codes en référence au questionnaire de l'annexe 1).

\section{Annex 2}

Contingency table of the answers, in number of departments (the codes refer to the questionnaire form of the annex 1 ).

\begin{tabular}{|c|c|c|c|c|c|c|c|c|c|c|c|c|c|c|c|}
\hline \multicolumn{8}{|c|}{ Nb de brigades où l'espèce est signalée } & \multicolumn{8}{|c|}{ Tendance 1995-2001 (code 2 annexe 1) } \\
\hline Année & ASA & APP & ASL & OCL & PFL & PCC & APT & $\mathrm{Nb} \mathrm{Dpt}$ & ASA & APP & ASL & $\mathrm{OCL}$ & PFL & PCC & APT \\
\hline 1977 & 6 & 68 & 0 & 70 & 0 & 0 & 0 & -- & 4 & 14 & 2 & 0 & 0 & 0 & \\
\hline 1990 & 21 & 73 & 27 & 84 & 21 & 10 & 0 & - & 2 & 26 & 4 & 15 & 1 & 4 & \\
\hline 1995 & 17 & 72 & 39 & 92 & 34 & 33 & 0 & 0 & 2 & 18 & 13 & 51 & 3 & 11 & 1 \\
\hline \multirow[t]{3}{*}{2001} & 16 & 73 & 46 & 93 & 61 & 49 & 1 & + & 2 & 2 & 3 & 12 & 19 & 11 & \\
\hline & & & & & & & & ++ & 0 & 5 & 9 & 8 & 30 & 14 & \\
\hline & & & & & & & & NSP & 6 & 8 & 15 & 9 & 8 & 9 & 4 \\
\hline \multicolumn{8}{|c|}{ Abondance $1^{\text {re }}$ cat. (code 3 annexe 1 ) } & \multicolumn{8}{|c|}{ Exploitation amateur $1^{\text {re }}$ cat. (code 4 annexe 1) } \\
\hline $\mathrm{Nb}$ Dpt & ASA & APP & ASL & OCL & PFL & PCC & APT & $\mathrm{Nb} \mathrm{Dpt}$ & ASA & APP & ASL & OCL & PFL & PCC & APT \\
\hline 0 & 5 & 2 & 20 & 27 & 5 & 23 & 1 & $0 / 1$ & 12 & 34 & 39 & 63 & 24 & 30 & 1 \\
\hline+ & 10 & 58 & 17 & 37 & 38 & 8 & 0 & + & 2 & 25 & 3 & 16 & 12 & 2 & 0 \\
\hline++ & 0 & 4 & 0 & 8 & 9 & 2 & 0 & ++ & 0 & 7 & 1 & 3 & 8 & 0 & 0 \\
\hline+++ & 0 & 2 & 0 & 3 & 1 & 0 & 0 & +++ & 0 & 2 & 0 & 0 & 8 & 0 & 0 \\
\hline NSP & 3 & 7 & 9 & 16 & 9 & 10 & 4 & NSP & 4 & 5 & 3 & 9 & 10 & 11 & 4 \\
\hline \multicolumn{8}{|c|}{ Abondance $2^{e}$ cat. (code 3 annexe 1 ) } & \multicolumn{8}{|c|}{ Exploitation amateur $2^{e}$ cat. (code 4 annexe 1 ) } \\
\hline $\mathrm{Nb} \mathrm{Dpt}$ & ASA & APP & ASL & OCL & PFL & PCC & APT & $\mathrm{Nb}$ Dpt & ASA & APP & ASL & $\mathrm{OCL}$ & PFL & PCC & APT \\
\hline 0 & 10 & 40 & 24 & 3 & 19 & 7 & 1 & $0 / 1$ & 26 & 56 & 34 & 21 & 25 & 14 & 0 \\
\hline+ & 3 & 25 & 14 & 15 & 21 & 15 & 0 & + & 0 & 10 & 5 & 54 & 12 & 12 & 0 \\
\hline++ & 0 & 1 & 0 & 37 & 6 & 9 & 0 & ++ & 0 & 1 & 1 & 9 & 8 & 4 & 0 \\
\hline+++ & 0 & 0 & 0 & 28 & 0 & 1 & 0 & +++ & 0 & 2 & 0 & 0 & 3 & 2 & 0 \\
\hline \multirow[t]{8}{*}{ NSP } & 5 & 7 & 8 & 8 & 16 & 11 & 4 & NSP & 2 & 6 & 6 & 7 & 14 & 11 & 5 \\
\hline & & & & & & & & \multicolumn{8}{|c|}{ Exploitation pro. $2^{\mathrm{e}}$ cat. (code 4 annexe 1 ) } \\
\hline & & & & & & & & $\mathrm{Nb} \mathrm{Dpt}$ & ASA & APP & ASL & OCL & PFL & PCC & APT \\
\hline & & & & & & & & $0 / 1$ & 15 & 69 & 40 & 67 & 47 & 31 & 1 \\
\hline & & & & & & & & + & 0 & 0 & 2 & 7 & 1 & 3 & 0 \\
\hline & & & & & & & & ++ & 0 & 0 & 0 & 2 & 0 & 0 & 0 \\
\hline & & & & & & & & +++ & 0 & 0 & 0 & 2 & 0 & 0 & 0 \\
\hline & & & & & & & & NSP & 3 & 4 & 4 & 13 & 14 & 9 & 4 \\
\hline \multicolumn{8}{|c|}{ Présence/Absence Plans d'eau publiques } & \multicolumn{8}{|c|}{ Présence/Absence Plans d'eau privés } \\
\hline $\mathrm{Nb}$ Dpt & ASA & APP & ASL & OCL & PFL & PCC & APT & $\mathrm{Nb}$ Dpt & ASA & APP & ASL & OCL & PFL & PCC & APT \\
\hline Absent & 16 & 71 & 44 & 41 & 53 & 36 & 5 & Absent & 9 & 69 & 28 & 52 & 23 & 25 & 5 \\
\hline Présent & 2 & 2 & 3 & 50 & 9 & 7 & 0 & Présent & 9 & 4 & 19 & 39 & 39 & 18 & 0 \\
\hline NSP & 0 & 0 & 0 & 0 & 0 & 0 & 0 & NSP & 0 & 0 & 0 & 0 & 0 & 0 & 0 \\
\hline \multicolumn{8}{|c|}{ Exploit. pro. Plans d'eau pub. (code 4 annexe 1) } & \multicolumn{8}{|c|}{ Exploit. amat. Plans d'eau pub. (code 4 annexe 1) } \\
\hline $\mathrm{Nb}$ Dpt & ASA & APP & ASL & OCL & PFL & PCC & APT & $\mathrm{Nb}$ Dpt & ASA & APP & ASL & $\mathrm{OCL}$ & PFL & PCC & APT \\
\hline $0 / I$ & 10 & 54 & 33 & 67 & 42 & 31 & 1 & $0 / 1$ & 9 & 52 & 31 & 40 & 34 & 27 & 1 \\
\hline+ & 0 & 0 & 0 & 2 & 1 & 0 & 0 & + & 0 & 2 & 2 & 27 & 5 & 2 & 0 \\
\hline++ & 0 & 0 & 0 & 2 & 0 & 0 & 0 & ++ & 1 & 0 & 0 & 3 & 2 & 0 & 0 \\
\hline+++ & 0 & 0 & 0 & 0 & 0 & 0 & 0 & +++ & 0 & 0 & 0 & 1 & 1 & 0 & 0 \\
\hline NSP & 8 & 19 & 14 & 20 & 19 & 12 & 4 & NSP & 8 & 19 & 14 & 20 & 20 & 14 & 4 \\
\hline
\end{tabular}

